\title{
FATTY ACIDS RELATED GENES EXPRESSION UNDERGO SUBSTANTIAL CHANGES IN PORCINE OVIDUCTAL EPITHELIAL CELLS DURING LONG-TERM PRIMARY CULTURE
}

Joanna Budna ${ }^{1}$, Piotr Celichowski ${ }^{1}$, Sandra Knap ${ }^{1}$, Maurycy Jankowski ${ }^{2}$, Magdalena Magas ${ }^{2}$, Mariusz J. Nawrocki ${ }^{2}$, Piotr Ramlau ${ }^{3}$, Andrzej Nowicki ${ }^{3}$, Magdalena Rojewska ${ }^{2}$, Błażej Chermuła ${ }^{4}$, Michal Jeseta $^{5}$, Paweł Antosik ${ }^{6}$, Dorota Bukowska ${ }^{6}$, Małgorzata Bruska $^{2}$, Maciej Zabel ${ }^{7,8}$, Michał Nowicki ${ }^{1}$, Bartosz Kempisty ${ }^{1,2,5 *}$

\begin{abstract}
The process of reproduction requires several factors, leading to successful fertilization of an oocyte by a single spermatozoon. One of them is the complete maturity of an oocyte, which is acquired during long stages of folliculogenesis and oogenesis. Additionally, the oviduct, composed of oviductal epithelial cells (OECs), has a prominent influence on this event through sperm modification and supporting oocyte's movement towards uterus.

OECs were isolated from porcine oviducts. Cells were kept in primary in vitro culture for 30 days. After 24h and on days 7, 15 and 30 cells were harvested, and RNA was isolated. Transcript changes were analyzed using microarrays. Fatty acids biosynthetic process and fatty acids transport ontology groups were selected for analysis and described.

Results of this study indicated that majority of genes in both ontology groups were up-regulated on day 7, 15 and 30 of primary in vitro culture. We analyzed genes involved in fatty acids biosynthetic process, including: GGT1, PTGES, INSIG1, SCD, ACSL3, FADS2, FADS1, ACSS2, ALOX5AP, ACADL, SYK, ACACA, HSD17B8, FADS3, OXSM, and transport, including: ABCC2, ACSL4, FABP3, PLA2G3, PPARA, SYK, PPARD, ACACA and P2RX7.

Elevated levels of fatty acids in bovine and human oviducts are known to reduce proliferation capacity of OECs and promote inflammatory responses in their microenvironment. Most of measured genes could not be connected to reproductive events. However, the alterations in cellular proliferation, differentiation and genes expression during in vitro long-term culture were significant. Thus, we can treat them as putative markers of changes in OECs physiology.
\end{abstract}

Running title: Fatty acids changes in porcine OECs during in vitro culture

Keywords: oviductal epithelial cells, fatty acids, microarray, pig

\footnotetext{
${ }^{1}$ Department of Histology and Embryology, Poznan University of Medical Sciences, Poznan, Poland

${ }^{2}$ Department of Anatomy, Poznan University of Medical Sciences, Poznan, Poland

${ }^{3}$ Department of Toxicology, Poznan University of Medical Sciences, Poznan, Poland

${ }^{4}$ Division of Infertility and Reproductive Endocrinology, Department of Gynecology, Obstetrics and Gynecological Oncology, Poznan University of Medical Sciences, Poznań, Poland

${ }^{5}$ Department of Obstetrics and Gynecology, University Hospital and Masaryk University, Jihlavská 20, 625 00 Brno, Czech Republic

${ }^{6}$ Veterinary Center, Nicolaus Copernicus University in Toruń, Torun, Poland

${ }^{7}$ Division of Histology and Embryology, Department of Human Morphology and Embryology, Wroclaw Medical University, Wroclaw, Poland

${ }^{8}$ Division of Anatomy and Histology, University of Zielona Góra, Zielona Góra, Poland

* Correspondence: bkempisty@ump.edu.pl

Full list of author information is available at the end of article
} 


\section{Introduction}

The process of reproduction requires several factors leading to successful fertilization of an oocyte by a single spermatozoon. One of them is complete maturity of an oocyte, which is acquired during long stages of folliculogenesis and oogenesis. Only properly matured MII oocyte can be fertilized, however each previous step impacts further embryo fate [1]. It is known that during cytoplasmic maturation oocytes stores large amounts of mRNA and proteins necessary for further embryo growth [2]. Our previous studies revealed large set of genes influencing maturation capability of porcine oocytes. We know that the differences in gene expression before and after in vitro maturation (IVM) reflect changes in oocytes and its susceptibility for future fertilization [3-5]. This observation can result in development of molecular diagnostic of female gametes before proceeding to IVM.

However, the quality of an oocyte is not an exclusive factor determining reproduction success. The oviduct has a prominent influence on this event through sperm modification and making it capable of fertilization. The sperm is deposited in diverse parts of female tract depending on the species. In pigs it is deposited in the cervix, however for fertilization it has to reach the oviduct through the utero-tubal junction [6]. Studies on modified mice showed that this passage is a critical step letting low percentage of spermatozoa to reach ampulla or the ampullar-isthmic junction. In spite of the fact that molecular basis of this event is still unknown, studies showed that lack of this transport results in infertility [7]. Furthermore, the oviduct. via process of adhesion. orchestrates oocyte's movement towards uterus, mainly attributed to cilia activity [8]. That is why events occurring during oviductal migration of cumulus-oocyte complexes (COCs) are so crucial.

To better understand biochemical changes governing oviductal journey of the COCs there is a need to bring the nature of oviductal epithelial cells (OECs) composing oviducts closer to understanding. For this purpose, we managed to keep OECs in longterm primary culture, resulting in biochemical and morphological changes in the cells. We have applied Affymetrix microarray assays to investigate expression profile of genes involved in several important processes of fatty acids biosynthesis and transport. Interest in this issue was caused by increasing number of reports describing detrimental effect of elevated non-esterified fatty acids (NEFA) levels on reproductive outcome. It may influence physiological functions of OECs, resulting in reduced OECs-sperm binding [9]. It was also hypothesized that raised fatty acid levels can lead to dysfunction and inflammation of OECs, causing infertility in bovine specimen [10].

\section{Material and Methods \\ Animals}

In this study, crossbred gilts $(n=45)$ at the age of about nine month and which displayed two regular estrous cycles were collected from a commercial herd. All the animals were checked daily for estrus behavior and were slaughtered after reaching the anestrus phase of the estrus cycle. The uteri were then transported to the laboratory within $30 \mathrm{~min}$ at $38^{\circ} \mathrm{C}$.

\section{Oviductal epithelial cells (OECs) selection and culture}

Oviducts were washed twice in Dulbecco's phosphate buffered saline (PBS) (137 mM NaCl, $27 \mathrm{mM}$ $\mathrm{KCl}, 10 \mathrm{mM} \mathrm{Na}_{2} \mathrm{HPO}_{4}, 2 \mathrm{mM} \mathrm{KH}_{2} \mathrm{PO}_{4}, \mathrm{pH}$ 7.4). Epithelial cells were surgically removed using sterile surgical blades. Then, the epithelium was incubated with collagenase I (Sigma Aldrich, Madison, USA), 1mg/ $\mathrm{mL}$ in Dulbecco's modified Eagle's medium (DMEM; Sigma Aldrich, Madison, USA) for $1 \mathrm{~h}$ at $37^{\circ} \mathrm{C}$. The cell suspension obtained from this digestion was filtered through $40 \mu \mathrm{m}$ pore size strainer to remove blood and single cells. The residue was collected by rinsing the strainer with DMEM. Cells were centrifuged (200 $\mathrm{x}$ g, 10 min.). Next, they were washed in PBS and centrifuged again. Later, they were incubated with $0.5 \%$ Trypsin/EDTA (Sigma Aldrich, Madison, USA) at $37^{\circ} \mathrm{C}$ for $10 \mathrm{~min}$. The reaction was stopped with fetal calf serum (FCS; Sigma Aldrich, Madison, USA). After incubation, cells where filtered and centrifuged for the last time. The final cell pellet was suspended in DMEM supplemented with $10 \%$ FCS, $100 \mathrm{U} / \mathrm{mL}$ penicillin, $100 \mu \mathrm{g} / \mathrm{mL}$ streptomycin and $1 \mu \mathrm{g} / \mathrm{mL}$ amphotericin $\mathrm{B}$. The cells were cultured at $37^{\circ} \mathrm{C}$ in a humidified atmosphere of $5 \% \mathrm{CO}_{2}$. Once the OEC cultures attained $70-80 \%$ confluency, they were passaged by washing with PBS, digested with $0.025 \%$ Trypsin/ EDTA, neutralized by a $0.0125 \%$ trypsin inhibitor (Cascade Biologics, Portland, USA), centrifuged, and resuspended at a seeding density of $2 \times 10^{4}$ cells $/ \mathrm{cm}^{2}$. The culture medium was changed every three days. The culture lasted 30 days.

\section{RNA extraction from oviductal epithelial cells (OECs)}

Oviductal epithelial cell were pooled and harvested 24h, 7 days, 15 days and 30 days after beginning of culture. Total RNA was extracted from samples using TRI Reagent (Sigma, St Louis, MO, USA) and RNeasy MinElute cleanup Kit (Qiagen, Hilden, Germany). The amount of total mRNA was determined from the optical density at $260 \mathrm{~nm}$, and the RNA purity was estimated using the $260 / 280 \mathrm{~nm}$ absorption ratio (higher than 1.8) (NanoDrop spectrophotometer, Thermo Scientific, ALAB, Poland). The RNA integrity and quality were checked on a Bioanalyzer 2100 (Agilent Technologies, Inc., Santa Clara, CA, USA). The resulting RNA integrity numbers (RINs) were between 8.5 and 10 with an average of 9.2 (Agilent Technologies, Inc., Santa Clara, CA, USA). The RNA in each sample was diluted to a concentration of $100 \mathrm{ng} / \mu \mathrm{l}$ with an OD260/OD280 ratio of 1.8/2.0. From each RNA sample, 100 ng of RNA was taken for microarray expression assays. 


\section{Microarray expression analysis and statistics}

Total RNA (100 ng) from each pooled sample was subjected to two rounds of sense cDNA amplification (Ambion® ${ }^{\circledR}$ WT Expression Kit). The obtained cDNA was used for biotin labeling and fragmentation by Affymetrix GeneChip ${ }^{\circledR}$ WT Terminal Labeling and Hybridization (Affymetrix). Biotin-labeled fragments of cDNA $(5.5 \mu \mathrm{g})$ were hybridized to the Affymetrix ${ }^{\circledR}$ Porcine Gene 1.1 ST Array Strip ( $48^{\circ} \mathrm{C} / 20 \mathrm{~h}$ ). Microarrays were then washed and stained according to the technical protocol using the Affymetrix GeneAtlas Fluidics Station. The array strips were scanned employing Imaging Station of the GeneAtlas System. Preliminary analysis of the scanned chips was performed using Affymetrix GeneAtlasTM Operating Software. The quality of gene expression data was confirmed according to the quality control criteria provided by the software. The obtained CEL files were imported into downstream data analysis software.

All of the presented analyses and graphs were performed using Bioconductor and R programming languages. Each CEL file was merged with a description file. In order to correct background, normalize, and summarize results, we used the Robust Multiarray Averaging (RMA) algorithm. To determine the statistical significance of the analyzed genes, moderated t-statistics from the empirical Bayes method were performed. The obtained p-value was corrected for multiple comparisons using Benjamini and Hochberg's false discovery rate. The selection of significantly altered genes was based on a p-value beneath 0.05 and expression higher than two-fold.

Differentially expressed genes were subjected selection by examination of genes involved in cell migration regulation. The differentially expressed gene list (separated for up- and down-regulated genes) was uploaded to DAVID software (Database for Annotation, Visualization and Integrated Discovery) [11].

Subsequently we analyzed the relation between the genes belonging to chosen GO terms with GOplot package [12]. The GoPlot package had calculated the z-score: the number of up-regulated genes minus the number of down-regulated genes divided by the square root of the count. This information allowed estimating the change course of each gene-ontology term.

Moreover, interactions between differentially expressed genes/proteins belonging to the chosen GO terms were investigated by STRING10 software (Search Tool for the Retrieval of Interacting Genes) [13]. The list of gene names was used as a query for an interaction prediction. The search criteria were based on co-occurrences of genes/proteins in scientific texts (text mining), co-expression, and experimentally observed interactions. The results of such analyses generated a gene/protein interaction network where the intensity of the edges reflected the strength of the interaction score.

Finally, the functional interaction between genes that belongs to the chosen GO BP terms were in- vestigated by REACTOME FIViz application to the Cytoscape 3.6.0 software. The ReactomeFIViz app is designed to find pathways and network patterns related to cancer and other types of diseases. This app accesses the pathways stored in the Reactome database, allowing to do pathway enrichment analysis for a set of genes, visualize hit pathways using manually laid-out pathway diagrams directly in Cytoscape, and investigate functional relationships among genes in hit pathways. The app can also access the Reactome Functional Interaction (FI) network, a highly reliable, manually curated pathway-based protein functional interaction network covering over $60 \%$ of human proteins.

\section{Ethical approval:}

The research related to animal use has been complied with all the relevant national regulations and institutional policies for the care and use of animals. Bioethical Committee approval no. 32/2012.

\section{Results}

To investigate oviductal epithelial cells' transcriptome changes following 7, 15 and 30 days after start of primary in vitro culture, we performed whole gene expression analysis by Affymetrix@ Porcine Gene 1.1 ST Array. In such assay expression of more than 14789 porcine transcripts was examined. The genes for which the fold change was higher than the cut-off value (fold $>|2|$ ) and corrected $p$ value $<0.05$, were considered as differentially expressed. From the whole transcript that consist of 2552 different genes. From these genes 1537 were up-regulated and 995 were down-regulated after 7 days of culture, 1471 were up-regulated and 1061 were down-regulated after 15 days of culture and 1329 were up-regulated and 1203 were down-regulated after 30 days of culture.

DAVID (Database for Annotation, Visualization and Integrated Discovery) software was used for extraction of the genes belong to "biological adhesion" gene ontology biological process term (GO BP). Up and down regulated gene sets were subjected to DAVID searching separately and only gene sets where adj. $\mathrm{p}$ value were lower than 0.05 were selected. The DAVID software analysis showed that differently expressed genes belongs to 657 Gene ontology Biological Process terms. In this paper we focused on "fatty acid biosynthetic process" and "fatty acid transport" GO BP terms. These sets of genes were subjected to hierarchical clusterization procedure and presented as heatmaps (Fig. 1). The gene symbols, fold changes in expression, Entrez gene IDs and corrected $p$ values of that genes were shown in Table 1. The enrichment of each GO BP term was calculated and shown on the circle diagram (Fig. 2).

Moreover, in Gene Ontology database genes that formed one particular GO group can also belong to other different GO term categories. By this reason we explore the gene intersections between selected GO BP terms. The relation between those GO BP 

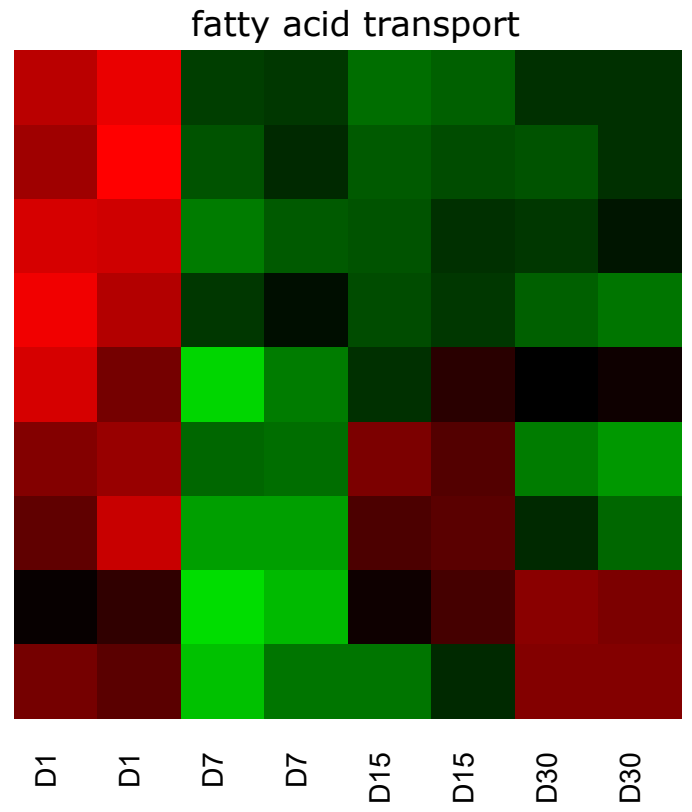
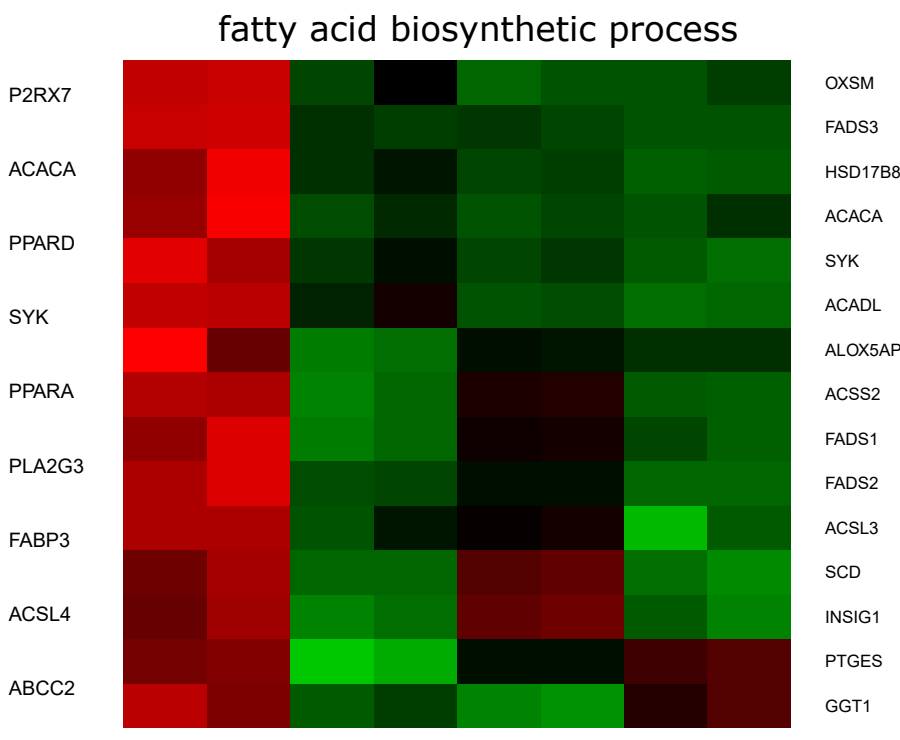

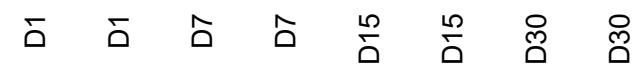

Figure 1 Heat map representation of differentially expressed genes belonging to the "fatty acid biosynthetic process" and "fatty acid transport" GO BP terms. Arbitrary signal intensity acquired from microarray analysis is represented by colours (green, higher; red, lower expression). Log2 signal intensity values for any single gene were resized to Row Z-Score scale (from -2, the lowest expression to +2 , the highest expression for single gene)

Table 1 Gene symbols, fold changes in expression of 7, 15 and 30 days vs. 1 day, corrected p values of 7, 15 and 30 days vs. 1 day, and Entrez gene IDs of studied genes

\begin{tabular}{|c|c|c|c|c|c|c|c|}
\hline Gene symbol & \begin{tabular}{|c|}
$\begin{array}{c}\text { Fold change } \\
\text { D1/D7 }\end{array}$ \\
\end{tabular} & $\begin{array}{c}\text { Fold change } \\
\text { D1/D15 }\end{array}$ & $\begin{array}{c}\text { Fold change } \\
\text { D1/D30 }\end{array}$ & $\begin{array}{c}\text { Corrected } p \text { values } \\
\text { D1/D7 }\end{array}$ & $\begin{array}{c}\text { Corrected p values } \\
\text { D1/D15 }\end{array}$ & $\begin{array}{c}\text { Corrected p values } \\
\text { D1/D30 }\end{array}$ & $\begin{array}{c}\text { Entrez gene } \\
\text { ID }\end{array}$ \\
\hline PTGES & 4.56368455 & 1.966507711 & 1.293063685 & $1.79 \mathrm{E}-05$ & 0.000459953 & 0.039606264 & 9536 \\
\hline ALOX5AP & 4.520547717 & 2.748499793 & 3.210201772 & 0.001121459 & 0.006556994 & 0.002543962 & 241 \\
\hline ACACA & 3.411749155 & 3.730045487 & 3.501387627 & 0.000587918 & 0.000306632 & 0.000278794 & 31 \\
\hline OXSM & 2.234415035 & 2.697009183 & 2.514316293 & 0.000560071 & 0.000142755 & 0.000144184 & 54995 \\
\hline PPARD & 9.200973366 & 6.826522496 & 5.602990807 & $2.25 \mathrm{E}-05$ & $2.24 \mathrm{E}-05$ & $2.49 \mathrm{E}-05$ & 5467 \\
\hline SYK & 3.464098651 & 4.018353552 & 4.895854715 & 0.000229841 & $9.08 \mathrm{E}-05$ & $2.95 \mathrm{E}-05$ & 6850 \\
\hline $\mathrm{ABCC} 2$ & 3.152758332 & 2.254643563 & 0.889698032 & 0.000681185 & 0.002954475 & 0.605678295 & 1244 \\
\hline $\mathrm{SCD}$ & 5.037882318 & 1.402767273 & 5.796847134 & $9.16 \mathrm{E}-05$ & 0.087483295 & $2.48 \mathrm{E}-05$ & 6319 \\
\hline $\mathrm{P} 2 \mathrm{RX7}$ & 6.165142343 & 8.522042354 & 5.795397835 & $2.69 \mathrm{E}-05$ & $6.72 \mathrm{E}-06$ & $1.03 \mathrm{E}-05$ & 5027 \\
\hline PLA2G3 & 2.632052041 & 1.157766391 & 2.959067511 & 0.000134655 & 0.251116813 & $3.17 \mathrm{E}-05$ & 50487 \\
\hline GGT1 & 2.150177795 & 2.663105611 & 1.37752522 & 0.0007667 & 0.000170914 & 0.032899596 & 2678 \\
\hline ACADL & 6.552573392 & 13.07544571 & 16.70384903 & $6.68 \mathrm{E}-05$ & $9.39 \mathrm{E}-06$ & $3.46 \mathrm{E}-06$ & 33 \\
\hline ACSL3 & 2.049555122 & 1.676911653 & 2.729972967 & 0.00209659 & 0.008605142 & 0.000218126 & 2181 \\
\hline ACSS2 & 6.349717983 & 2.503393425 & 5.620929728 & $7.45 \mathrm{E}-06$ & $9.49 \mathrm{E}-05$ & $2.39 \mathrm{E}-06$ & 55902 \\
\hline INSIG1 & 3.926907199 & 1.159777508 & 3.622271082 & 0.000131683 & 0.423799946 & 7.76E-05 & 3638 \\
\hline FADS2 & 5.435444175 & 3.800474915 & 6.759124087 & $2.43 E-05$ & $3.97 \mathrm{E}-05$ & $4.31 \mathrm{E}-06$ & 9415 \\
\hline FADS1 & 3.584889168 & 2.04605082 & 3.123560576 & 0.000163335 & 0.0020277 & 0.000134856 & 3992 \\
\hline FADS3 & 9.516455014 & 10.25018918 & 12.30064196 & 4.27E-06 & $1.47 \mathrm{E}-06$ & $4.44 \mathrm{E}-07$ & 3995 \\
\hline PPARA & 2.156741413 & 1.463696693 & 1.431759934 & 0.00169711 & 0.036412442 & 0.040484399 & 5465 \\
\hline $\mathrm{FABP} 3$ & 1.631820824 & 0.86444541 & 1.752248928 & 0.100739694 & 0.655180389 & 0.053056158 & 2170 \\
\hline HSD17B8 & 2.541991425 & 2.924133173 & 3.27902175 & 0.001028516 & 0.00039817 & 0.000167536 & 7923 \\
\hline ACSL4 & 2.078474506 & 0.952525669 & 0.719494326 & 0.000888279 & 0.778846768 & 0.027678635 & 2182 \\
\hline
\end{tabular}




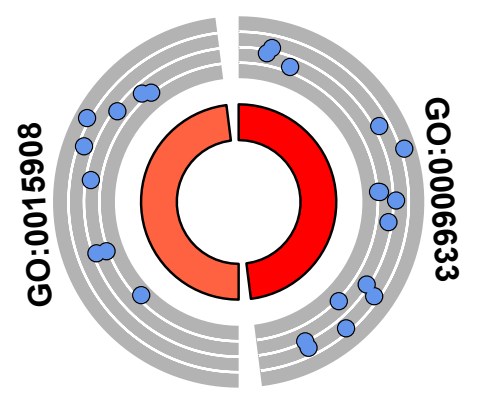

\begin{tabular}{|c|c|}
\hline ID & Description \\
\hline GO:0006633 & fatty acid biosynthetic process \\
\hline GO:0015908 & fatty acid transport \\
\hline
\end{tabular}

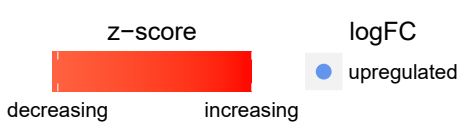

Figure 2 The circle plot showing the differently expressed genes and z-score of "fatty acid biosynthetic process" and "fatty acid transport" GO BP terms. The outer circle shows a scatter plot for each term of the fold change of the assigned genes. Points display logarithm from gene expression of up-regulated and down-regulated genes. The inner circle shows the z-score of each GO BP term. The width of the each bar corresponds to the number of genes within GO BP term and the color corresponds to the z-score

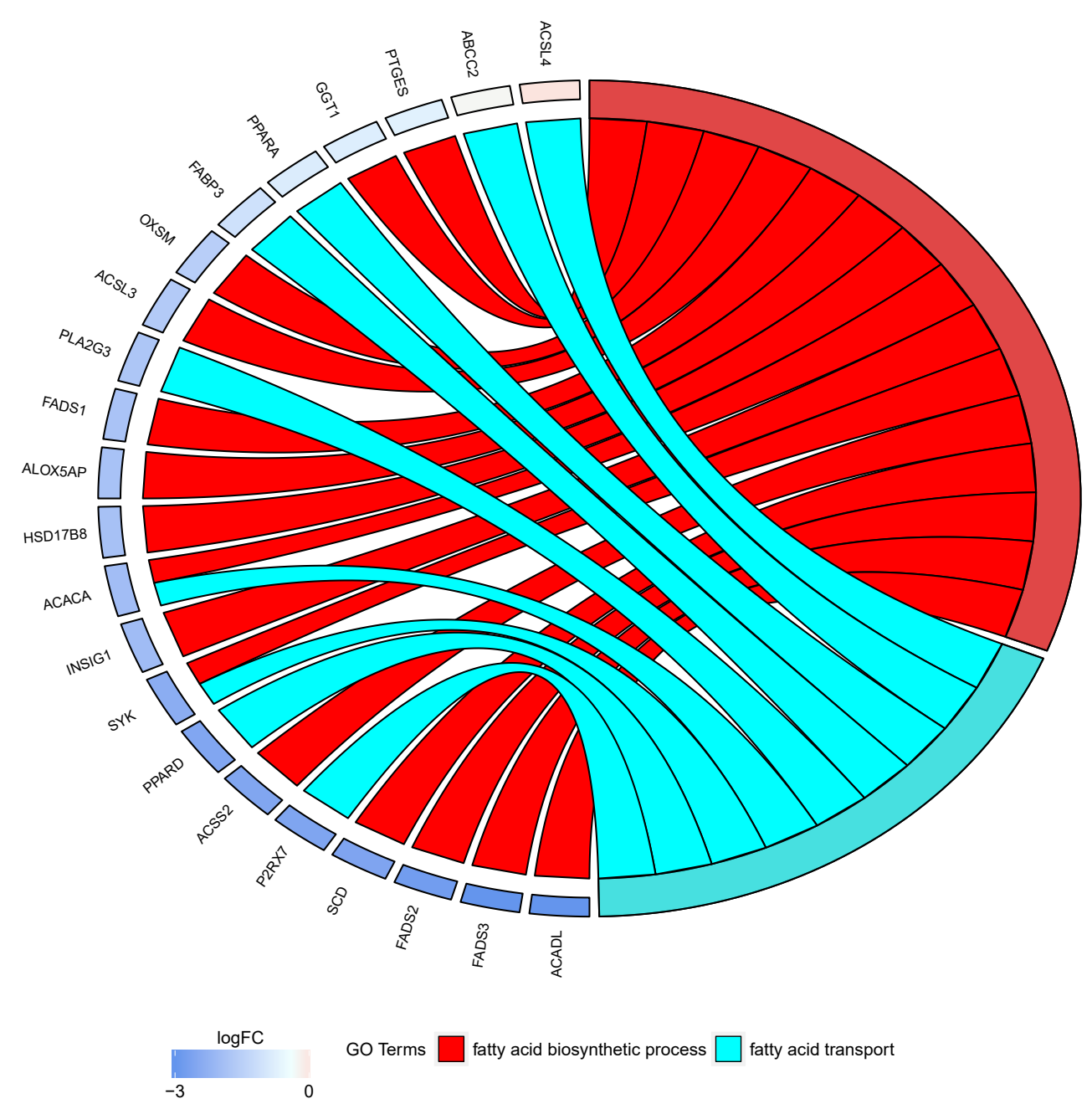

Figure 3 The representation of the mutual relationship between "fatty acid biosynthetic process" and "fatty acid transport" GO BP terms. The ribbons indicate which gene belongs to which categories. The genes were sorted by logFC from most to least changed gene 
terms was presented as circle plot (Fig. 3) as well as heatmap (Fig. 4). STRING-generated interaction network among differentially expressed genes belonging to each of selected GO BP terms. Using such prediction method provided us molecular interac- tion network formed between protein products of studied genes (Fig. 5). Finally, we investigated the functional interactions between chosen genes with REACTOME FIViz app to Cytoscape 3.6.0 software. The results were shown in Figure 6.

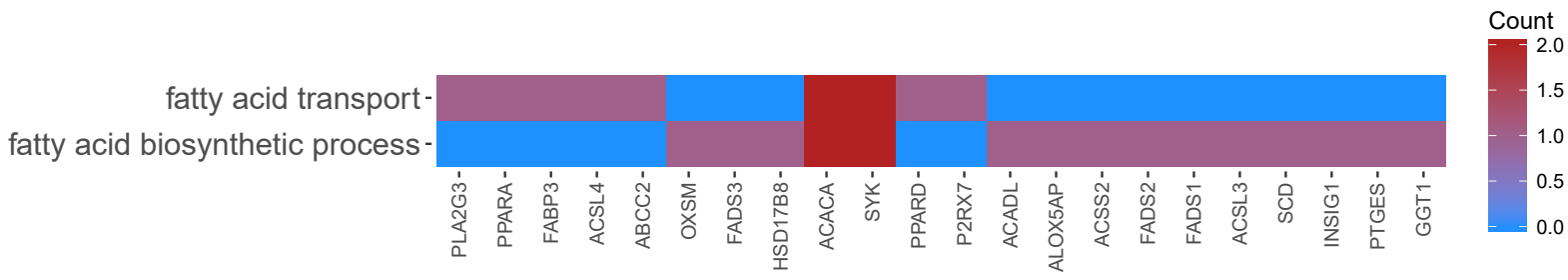

Figure 4 Heatmap showing the gene occurrence "fatty acid biosynthetic process" and "fatty acid transport" GO BP terms

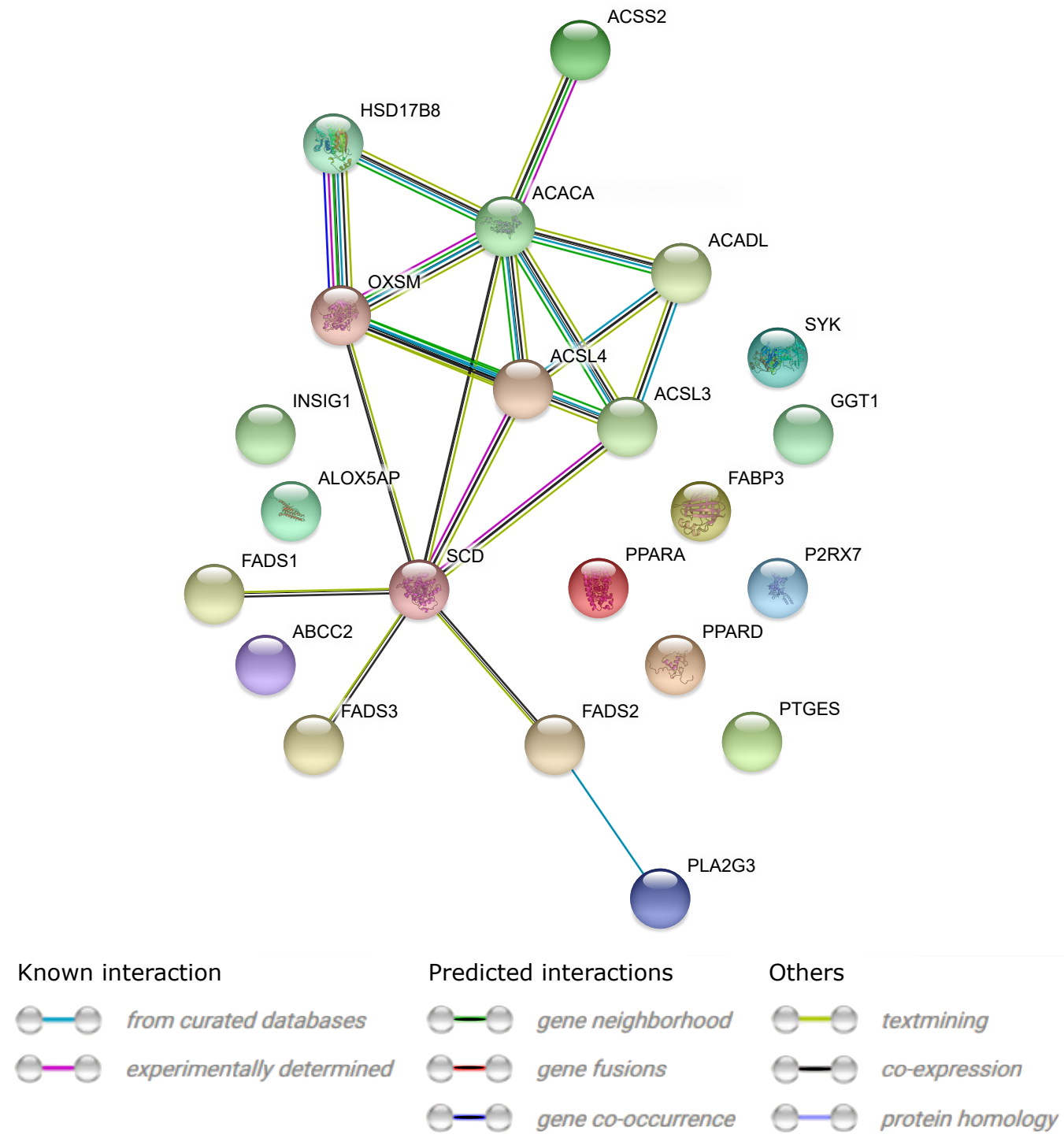

Figure 5 STRING-generated interaction network among differentially expressed genes belonging to the "fatty acid biosynthetic process" and "fatty acid transport" GO BP terms. The intensity of the edges reflects the strength of interaction score 


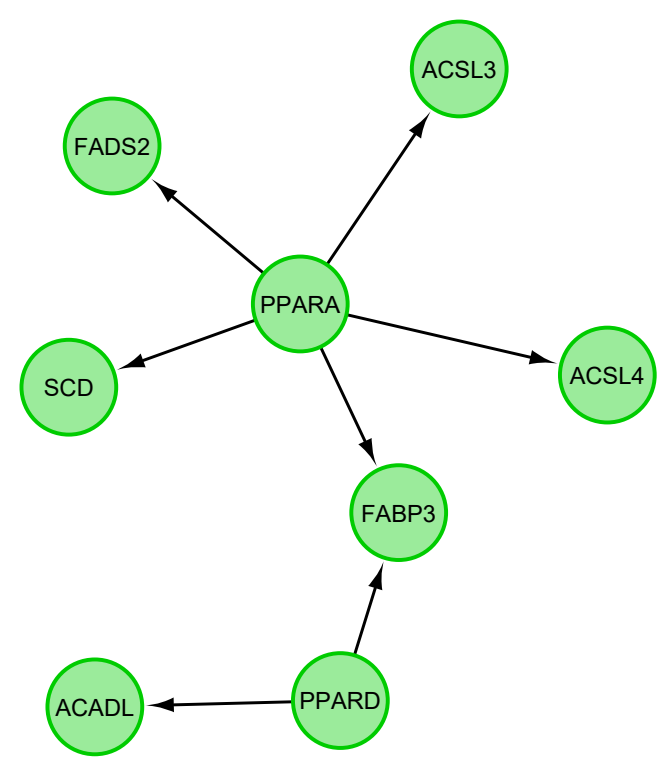

Figure 6 Functional interaction (FI) between differentially expressed genes belonging to the "fatty acid biosynthetic process" and "fatty acid transport" GO BP terms. In following figure "->" stands for activating/catalyzing, "-|" for inhibition, "-" for FIs extracted from complexes or inputs, and "---" for predicted FIs

\section{Discussion}

It was shown in cows that elevated concentration of non-esterified fatty acids (NEFA) in plasma are probably caused by intensified release from adipose tissue [14]. Among many others, metabolic disorders have been attributed to high levels of NEFA [15]. Importantly, increase in NEFA concentration in bovine and human ovarian follicles [16] was found to impair reproductive events, such as: oocyte maturation, fertilization, cleavage rate and quality of blastocyst [17]. Additionally, elevated levels of fatty acids can negatively influence ovarian granulosa and theca cells proliferation and steroidogenesis [18]. There are 5 types of fatty acids recently identified in oviductal tract: palmictic acid (PA), stearic acid, oleic acid, linoleic acid and myristic acid [19]. Even though they were identified in hen, there are also reports describing their role in mammalian oviducts. In bovine specimen and human, NEFA was shown to reduce proliferation capacity of OECs [20] and promote inflammatory responses in their microenvironment [21].

To investigate fatty acids changes and administration in porcine OECs long-term culture we analyzed expression level of genes involved in fatty acids biosynthetic process, including: GGT1, PTGES, INSIG1, SCD, ACSL3, FADS2, FADS1, ACSS2, ALOX5AP, ACADL, SYK, ACACA, HSD17B8, FADS3, OXSM and transport, including: ABCC2, ACSL4, FABP3, PLA2G3, PPARA, SYK, PPARD, ACACA and P2RX7.

The overall trend in both groups was an increase in genes expression after first day of culture, maintaining in comparable level till day 30 of culture. All genes were related to fatty acids homeostasis, however majority of them has not been previously related to OECs biology or other reproductive processes. We managed to link only some of them with reproduction, including oviductal tract physiology. Stearoyl-CoA desaturase (SCD) and fatty acid desaturase 2 (FADS2) are enzymes belonging to fatty acid desaturases family. While the first one is predominantly engaged in oleic acid biosynthesis, the second regulates unsaturation of fatty acids by creating double bonds between defined carbons of the fatty acyl chain. Studies including both genes were performed in order to test the influence of fatty acids supplementation before in vitro-derived ewe embryos freezing on further embryo quality. Results showed that prior fatty acids administration down-regulated $S C D 1$ and FADS2 expression on day 6 in embryos cultured in standard medium, co-cultured with bovine OECs and developing in ewe oviduct. This can indicate the imbalance of unsaturated/saturated fatty acids, possibly leading to changes in membrane fluidity [22].

Another genes - acyl-CoA synthetase long chain family member 3 (ACSL3) and insulin-induced gene 1 (INSIG1) were analyzed in oocytes and surrounding cumulus cells, respectively. The ACSL3 converts free long-chain fatty acids into fatty acyl-CoA esters, taking part in synthesis of lipids and fatty acids degradation. Studies in Xenopus Laevis frogs showed that loss of ACSL family members activity can lead to premature ovarian failure (POF) by acceleration of oocytes maturation and impaired meiotic arrest [23]. In turn, INSIG1 was analyzed in relation to follicle-stimulating hormone (FSH), LH (luteinizing hormone) and insulin administration. It was found that high insulin concentration up-regulated levels of INSIG1 mRNA, showing that physiological con- 
centrations of hormones are more likely to mirror in vivo outcome [24].

Among genes related to fatty acid transport some were already found in mammalian reproductive tract. A few of them, like fatty acid binding protein 3 (FABP3), acyl-CoA synthetase long chain family member (ACSL), and acetyl-CoA carboxylase alpha (ACACA) were analyzed in bovine OECs. The aim of the study was to examine influence of elevated NEFA concentrations on bovine OECs physiology, represented by metabolism and barrier function. Thus, expression levels of genes implicated in OECs viability, oxidative stress, carbohydrate and lipid metabolism was measured. It was found that expression of $F A B P 3$ and $A C S L$ genes was up-regulated, while $A C A$ $C A$ gene presented down-regulated expression. This let the conclusion, that increased levels of NEFA have detrimental effects on OECs metabolism and their barrier function. However the oviduct has the ability to modulate its microenvironment by tempering possible lipotoxic effects, thus favoring early embryo quality and survival [25].

ATP-binding cassette subfamily $\mathrm{C}$ member 2 (ABCC2) is a protein from family of $A B C$ transporters involved in multi-drug resistance. Presumably, $\mathrm{ABC}$ transporters play some role in gametogenesis and folliculogenesis, by involvement in steroids administration, and fetal development [26]. Molecular and histological evaluation presented the evidence that ABCC2 transporter is present in caprine ovarian tissue [27]. This does not exclude possibility of its presence in porcine ovaries and oviducts as well.

Summing up, most of measured genes could not be found in reports describing reproductive events. However, the alterations in cellular proliferation, differentiation and genes expression during in vitro long-term cell culture were significant. Thus, we can treat them as putative markers of changes in OECs physiology. Additionally, our primary in vitro cell culture provides information about OECs-dependent specificity and plasticity, however the physiological and molecular properties of these cells still need further studies.

\section{Acknowledgements}

Publication of this article was made possible by grants number 2014/13/D/NZ9/04798 "SONATA" and UMO-2012/07/N/ NZ5/00069 from Polish National Centre of Science

\section{Corresponding author}

Bartosz Kempisty PhD, Department of Histology and Embryology, Department of Anatomy, Poznań University of Medical Sciences, 6 Święcickiego St., 60-781 Poznań, Poland, phone/fax: +4861 8546418/+48618546440, e-mail: bkempisty@ump.edu.pl

\section{Conflict of interest statement}

The authors declare they have no conflict of interest

\section{References}

1. Orisaka M, Tajima K, Tsang BK, Kotsuji F. Oocyte-granulosa-theca cell interactions during preantral follicular development. J Ovarian Res. 2009;2(1):9; DOI:10.1186/1757-2215-2-9.
2. Eppig JJ. Coordination of nuclear and cytoplasmic oocyte maturation in eutherian mammals. Reprod Fertil Dev. 1996;8(4):485-489; DOI:10.1071/RD9960485

3. Budna J, Bryja A, Celichowski P, Kahan R, Kranc W, Ciesiółka S, Rybska M, Borys S, Jeseta M, Bukowska D, Antosik P, Brüssow KP, Bruska M, Nowicki M, Zabel M, Kempisty B. Genes of cellular components of morphogenesis in porcine oocytes before and after IVM. Reproduction. 2017;154(4):535-545, DOI:10.1530/REP-17-0367.

4. Budna J, Rybska M, Ciesiółka S, Bryja A, Borys S, Kranc W, Wojtanowicz-Markiewicz K, Jeseta M, Sumelka E, Bukowska D, Antosik P, Brüssow KP, Bruska M, Nowicki M, Zabel M, Kempisty B. Expression of genes associated with BMP signaling pathway in porcine oocytes before and after IVM - a microarray approach. Reprod Biol Endocrinol. 2017;15(1):43; DOI:10.1186/s12958-017-0261-6.

5. Kranc W, Budna J, Chachuła A, Borys S, Bryja A, Rybska M, Ciesiółka S, Sumelka E, Jeseta M, Brüssow KP, Bukowska D, Antosik P, Bruska M, Nowicki M, Zabel M, Kempisty B. Cell Migration is the ontology group differentially expressed in porcine oocytes before and after in vitro maturation: a microarray approach. DNA Cell Biol. 2017;36(4):273-282; DOI:10.1089/dna.2016.3425

6. Coy P, García-Vázquez FA, Visconti PE, Avilés M. Roles of the oviduct in mammalian fertilization. Reproduction. $\overline{2012 ; 1} \overline{44} \overline{(6)}: \overline{649-60}$; DOI:10.1530/REP-12-0279.

7. Tokuhiro K, Ikawa M, Benham AM, Okabe M. Protein disulfide isomerase homolog PDILT is required for quality control of sperm membrane protein ADAM3 and male fertility. Proc Natl Acad Sci USA. 2012;109(10):3850-3855; DOI:10.1073/pnas.1117963109.

8. Kölle S, Dubielzig S, Reese S, Wehrend A, König P, Kummer W. Ciliary transport, gamete interaction, and effects of the early embryo in the oviduct: ex vivo analyses using a new digital videomicroscopic system in the cow. Biol Reprod. 2009;81(2):267-74; DOI:10.1095/ biolreprod.108.073874.

9. Jordaens L, Arias-Alvarez M, Pintelon I, Thys S, Valckx S, Dezhkam Y, Bols PE, Leroy JL. Elevated non-esterified fatty acid concentrations hamper bovine oviductal epithelial cell physiology in three different in vitro culture systems. Theriogenology. 2015;84(6):899-910; DOI:10.1016/j. theriogenology.2015.05.030.

10. Ohtsu, A, Tanaka H, Seno K, Iwata H, Kuwayama T, Shirasuna K. Palmitic acid stimulates interleukin-8 via the TLR4/NF-B/ROS pathway and in duces mitochondrial dysfunction in bovine oviduct epithelial cells. Am J Reprod Immunol. 2017;77(6); DOI:10.1111/aji.12642.

11. Huang DW, Sherman BT, Tan Q, Collins JR, Alvord WG, Roayaei J, Stephens R, Baseler MW, Lane HC, Lempicki RA. The DAVID Gene Functional Classification Tool: a novel biological module-centric algorithm to functionally analyze large gene lists. Genome Biol. 2007;8(9):R183; DOI:10.1186/gb-2007-8-9-r183.

12. Wencke W, Sánchez-Cabo F, Ricote M. GOplot: an R package for visually combining expression data with functional analysis. Bioinformatics. 2015;31(17):2912-4; DOI:10.1093/bioinformatics/btv300.

13. von Mering C, Jensen LJ, Snel B, Hooper SD, Krupp M, Foglierini M, Jouffre N, Huynen MA, Bork P. STRING: known and predicted protein-protein associations, integrated and transferred across organisms. Nucleic Acids Res. 2005;33(database issue):D433-7; DOI:10.1093/nar/gki005.

14. Kawashima C, Matsui M, Shimizu T, Kida K, Miyamoto A. Nutritional factors that regulate ovulation of the dominant follicle during the first follicular wave postpartum in high-producing dairy cows. J Reprod Dev. 2012;58(1):10-16; DOI:10.1262/jrd.11-139N.

15. LeBlanc SJ. Interactions of metabolism, inflammation, and reproductive tract health in the postpartum period in dairy cattle. Reprod Domest Anim.2012;47 Suppl 5:18-30; DOI:10.1111/j.1439-0531.2012.02109.x.

16. Jungheim ES, Macones GA, Odem RR, Patterson BW, Lanzendorf SE, Ratts VS, Moley KH. Associations between free fatty acids, cumulus oocyte complex morphology and ovarian function during in vitro fertilization. Fertil Steril. 2011;95(6):1970-1974; DOI:10.1016/j.fertnstert.2011.01.154. Epub 2011 Feb 26.

17. Van Hoeck, V, Sturmey RG, Bermejo-Alvarez P, Rizos D, Gutierrez-Adan A, Leese HJ, Bols PE, Leroy JL. Elevated non-esterified fatty acid concentrations during bovine oocyte maturation compromise early embryo physiology. PLoS One. 2011;6(8):e23183; DOI:10.1371/journal. pone.0023183.

18. Vanholder, T, Leroy JL, Soom AV, Opsomer G, Maes D, Coryn M, de Kruif A Effect of non-esterified fatty acids on bovine granulosa cell steroidogenesis and proliferation in vitro. Anim Reprod Sci. 2005;87(1-2):33-44; DOI:10.1016/j.anireprosci.2004.09.006

19. Huang A, Isobe N, Obitsu T, Yoshimura Y. Expression of lipases and lipid receptors in sperm storage tubules and possible role of fatty acids in sperm survival in the hen oviduct. Theriogenology. 2016;85(7):13341342; DOI:10.1016/j.theriogenology.2015.12.020. 
20. Jordaens L, Arias-Alvarez M, Pintelon I, Thys S, Valckx S, Dezhkam Y, Bols PE, Leroy JL. Elevated non-esterified fatty acid concentrations hamper bovine oviductal epithelial cell physiology in three different in vitro culture systems. Theriogenology. 2015;84(6):899-910; DOI:10.1016/j. theriogenology.2015.05.030.

21. Wen H, Gris D, Lei Y, Jha S, Zhang L, Huang MT, Brickey WJ, Ting JP. Fatty acid-induced NLRP3-ASC inflammasome activation interferes with insulin signaling. Nat Immunol. 2011;12(5):408-415; DOI:10.1038/ni.2022.

22. Al Darwich, A, Perreau C, Petit MH, Papillier P, Dupont J, Guillaume D, Mermillod P, Guignot F. Effect of PUFA on embryo cryoresistance, gene expression and AMPKalpha phosphorylation in IVF-derived bovine embryos. Prostaglandins Other Lipid Mediat. 2010;93(1-2):30-6; DOI:10.1016/j.prostaglandins.2010.06.002.

23. Wang HW, Fang JS, Kuang X, Miao LY, Wang C, Xia GL, King ML, Zhang J. Activiy of long-chain acyl-CoA synthetase is required for maintaining meiotic arrest in Xenopus laevis. Biol Reprod. 2012;87(3):74; DOI:10.1095/biolreprod.112.100511.

24. Sanchez F, Romero S, Smitz J. Oocyte and cumulus cell transcripts from cultured mouse follicles are induced to deviate from normal in vivo conditions by combinations of insulin, follicle-stimulating hormone, and human chorionic gonadotropin. Biol Reprod. 2011;85(3):565-74, DOI:10.1095/biolreprod.111.091744.

25. Jordaens L, Van Hoeck V, Maillo V, Gutierrez-Adan A, Marei WFA, Vlaeminck B, Thys S, Sturmey RG, Bols PEJ, Leroy JLMR. Maternal metabolic stress may affect oviduct gatekeeper function. Reproduction. 2017;153(6):759-773; DOI:10.1530/REP-16-0569.

26. Wang H, Zhou L, Gupta A, Vethanayagam RR, Zhang Y, Unadkat JD, Mao Q. Regulation of BCRP/ABCG2 expression by progesterone and 17beta-estradiol in human placental BeWo cells. Am J Physiol Endocrinol. 2006;290(5):e798-807; DOI:10.1152/ajpendo.00397.2005

27. Guerreiro DD, de Lima LF, Mbemya GT, Maside CM, Miranda AM, Tavares KCS, Alves BG, Faustino LR, Smitz J, de Figueiredo JR, Rodrigues APR ATP-binding cassette $(\mathrm{ABC})$ transporters in caprine preantral follicles: gene and protein expression. Cell Tissue Res. 2018;372(3):611-620; DOI:10.1007/s00441-018-2804-3. 